\title{
Prevalence and prognosis of stress disorders: a review of the epidemiologic literature
}

This article was published in the following Dove Press journal:

Clinical Epidemiology

3 May 2017

Number of times this article has been viewed

\author{
Jaimie L Gradus ${ }^{1-4}$ \\ 'National Center for PTSD, VA Boston \\ Healthcare System, ${ }^{2}$ Department \\ of Psychiatry, Boston University \\ School of Medicine, ${ }^{3}$ Department \\ of Epidemiology, Boston University \\ School of Public Health, Boston, \\ MA, USA; ${ }^{4}$ Department of Clinical \\ Epidemiology, Aarhus University \\ Hospital, Aarhus, Denmark
}

\begin{abstract}
Given the ubiquity of traumatic events, it is not surprising that posttraumatic stress disorder (PTSD) - a common diagnosis following one of these experiences - is characterized as conferring a large burden for individuals and society. Although there is recognition of the importance of PTSD diagnoses throughout psychiatry, the literature on other diagnoses one may receive following a stressful or traumatic event is scant. This review summarizes the literature on stress disorders (classified according to the International Classification of Diseases, 10th Edition [ICD-10]), including acute stress reaction, PTSD, adjustment disorder and unspecified stress reactions. This review focuses on the literature related to common psychiatric and somatic consequences of these disorders. The prevalence and incidence of each disorder are described A review of epidemiologic studies on comorbid mental health conditions, including depression, anxiety and substance abuse, is included, as well as a review of epidemiologic studies on somatic outcomes, including cancer, cardiovascular disease and gastrointestinal disorders. Finally, the current literature on all-cause mortality and suicide following stress disorder diagnoses is reviewed. Stress disorders are a critical public health issue with potentially deleterious outcomes that have a significant impact on those living with these disorders, the health care system and society. It is only through an awareness of the impact of stress disorders that appropriate resources can be allocated to prevention and treatment. Future research should expand the work done to date beyond the examination of PTSD, so that the field may obtain a more complete picture of the impact all stress disorders have on the many people living with these diagnoses.
\end{abstract}

Keywords: trauma- and stressor-related disorders, stress disorders, traumatic, prognosis

\section{Introduction}

Stress diagnoses are receiving attention due to recent increases in traumatic events (e.g., conflict, terrorism, natural disasters). A trauma is an event in which a person is exposed to actual or threatened death, serious injury or sexual violation. ${ }^{1}$ Traumatic experiences are common; it is estimated that $50 \%$ of adults will experience a traumatic event in their lifetime..$^{2-4}$ Given the ubiquity of traumatic events, it is not surprising that posttraumatic stress disorder (PTSD) - a common diagnosis following one of these experiences - is characterized as conferring a large burden for individuals and society. ${ }^{5}$

Although there is recognition of the importance of PTSD diagnoses throughout psychiatry, the literature on other diagnoses one may receive following a stressful or traumatic event is scant. The goal of this review is to summarize the literature on important potential outcomes of stress disorders (classified according to the International Classification of Diseases, 10th Edition [ICD-10]), including psychiatric and somatic sequelae that have been frequently highlighted in the literature. ${ }^{6}$ First, the definition of
Correspondence: Jaimie L Gradus National Center for PTSD, VA Boston Healthcare System, I50 S. Huntington Avenue, II6B-3, Boston, MA 02130, USA Tel +l 8573646688 Email jaimie.gradus@va.gov 
each stress disorder included in ICD-10 is summarized and what is known about their prevalence is described. Next, the literature on common deleterious mental and physical health sequelae of stress disorders is reviewed, as well as mortality. Finally, conclusions and recommendations for future research are discussed.

PubMed was used to search for English-language articles on epidemiological, population-based studies of adults published between January 1, 1994, and December 31, 2016, for inclusion in this review. Search terms included "acute stress reaction", "posttraumatic stress disorder", "adjustment disorder" and "unspecified reaction to stress" individually and in combination with the following terms: "depression", "anxiety", "alcohol", "drug use”, "cancer", "cardiovascular", "gastrointestinal", "all-cause mortality" and "suicide". The original searches for "posttraumatic stress disorder" and "adjustment disorder" individually returned almost 30,000 articles published during the review period; hence, the additional qualifiers of "epidemiology" and "prognosis" were added to all searches that included these terms. Studies were excluded from the review if they were returned in error (e.g., not on the topic of the search), not epidemiological or population based (e.g., treatment studies, studies using small clinical samples) or examined an outcome that is outside the scope of this review (e.g., stress disorders as an outcome of trauma or physical illness). This search yielded 1,021 results, of which we included 43 studies based on the abovementioned criteria (Figure 1).

\section{Definitions of stress disorders}

There are five diagnoses within ICD-10 that can be given following a stressful or traumatic event from which one is having difficulty recovering. These include acute stress reaction (a diagnosis given in the immediate aftermath of the event), PTSD (a diagnosis given following a traumatic event and a timed period of non-recovery), adjustment disorder (a diagnosis given after a stressful event and a timed period of non-recovery) and two catch-all diagnoses, other reactions to severe stress and reactions to severe stress unspecified (given to people who are experiencing distress following a stressful or traumatic event but who do not meet the full diagnostic criteria for one of the other disorders).

The evolution in diagnostic categories across ICD versions is reflective of the evolution of the field of psychiatry's thinking regarding stress diagnoses themselves over the last half century. Severe stress and trauma have always been a

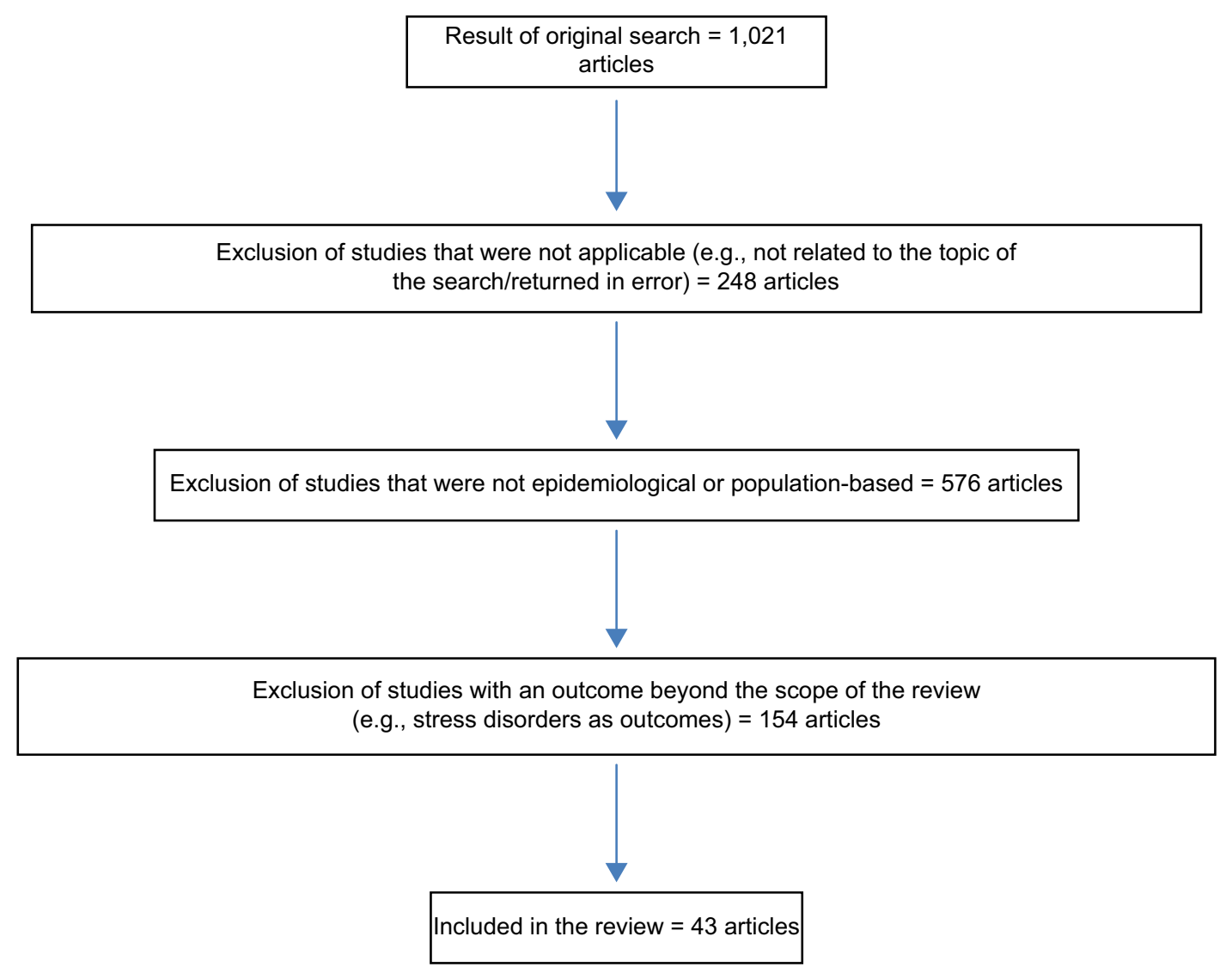

Figure I Flow chart of articles published between January I, 1994, and December 3I, 2016, and reviewed for inclusion. 
part of the human condition. But it was not until after the first and second World Wars that increased attention was paid to the psychiatric consequences of trauma. ${ }^{7}$ Since then, our understanding of posttrauma psychopathology has continually evolved into the diagnostic categorizations we use today.

Stress disorders were first introduced as a diagnostic category in ICD-10.6,8 Prior to that, in ICD-9, only acute stress reaction and adjustment disorder were included as diagnoses, and these were subsumed under a category of “other" adult-onset diagnoses. ${ }^{9}$ As the diagnosis of PTSD as we know it today was not formulated until 1980, this diagnosis was not included in ICD-8. Instead, a stress diagnosis of "transient situational disturbance" was included in ICD-8, which encompassed all stress reactions, including adjustment issues and stress and reactions to combat. ${ }^{9}$ Currently, ICD-10 stress diagnoses are among the most frequently assigned psychiatric diagnoses worldwide. ${ }^{9}$ Each is described in more detail below.

Acute stress reaction (ICD-10 code: F43.0) is a short-term diagnosis given when symptomatology appears within 1 hour of the occurrence of a severe stress or traumatic event (e.g., natural disaster, combat, severe accident, witnessing violent death, sexual assault), with symptoms beginning to abate within 48 hours. ${ }^{6}$ Besides an experience of a traumatic event, diagnostic criteria for acute stress reaction include the possible experience of transient depression, anxiety, "daze"-like, anger, despair, overactivity and social withdrawal symptoms, with no one symptom being dominant. Patients who receive this diagnosis must have no other mental disorder that could account for this symptomatology.

PTSD (ICD-10 code: F43.1) is a severe and chronic disorder that is diagnosed after the experience of a severe stress or traumatic event (e.g., natural disaster, combat, severe accident, witnessing violent death, sexual assault). ${ }^{6}$ According to ICD-10 criteria, PTSD symptomatology must be present within 6 months after the traumatic experience for a diagnosis to be given. Symptoms of PTSD, which in some cases may last for decades, include persistent reliving of the event through nightmares or flashbacks, avoidance of reminders of the event, inability to recall part of the event and a general state of hyperarousal (e.g., difficulty sleeping or concentrating, exaggerated startle response).

Adjustment disorder (ICD-10 code: F43.2) is a diagnosis given following a major psychosocial stressor or life event (e.g., divorce, job loss, serious physical illness) with symptom onset within 1 month of the event and with symptoms generally abating by 6 months after the event. ${ }^{6}$ Symptoms of adjustment disorder are similar to those of affective disorders (e.g., depression, anxiety, conduct or emotional disturbance), but a diagnosis of adjustment disorder can be given if an identifiable psychosocial stressor was experienced and the criteria for a diagnosis of a specific affective disorder are not met.

Two unspecified catch-all diagnoses can also be given following a severe stress or traumatic event, which are other reactions to severe stress (ICD-10 code: F43.8) and reactions to severe stress, unspecified (ICD-10 code: F43.9). ${ }^{6}$ Diagnoses of this type are typically reserved for patients who have experienced a stressful or traumatic event and who are experiencing distressing symptomatology but do demonstrate the diagnostic symptom criteria required to receive one of the stress diagnoses listed earlier (e.g., patients with subsyndromal stress disorders).

\section{Cross-national considerations in disorder definitions}

Although the ICD is used throughout most of the world for diagnosis, for mental disorders another diagnostic guide is also used, primarily in the US, called the Diagnostic and Statistical Manual for Mental Disorders (DSM). ${ }^{1}$ It is important to consider the differences in diagnostic classification between the ICD and DSM when thinking about the generalizability of results presented within this review, as studies conducted within the United States would have primarily used DSM diagnostic criteria. Importantly, a study that examined the concordance between ICD-10 and DSM diagnostic criteria for PTSD found that the agreement between the two diagnostic systems was only fair $($ kappa $=0.50)$ with about half of discrepancies arising from the additional DSM criteria of clinically significant distress. ${ }^{10}$

\section{Prevalence of stress disorders}

Research on the prevalence and incidence of stress disorders is limited. Of the stress disorders, only the prevalence of PTSD has been estimated in multiple general population studies, and much of this work has been conducted in the US where the $D S M$ is used for diagnoses, rather than ICD. In the most widely cited studies of the prevalence of PTSD, based on data from the National Comorbidity Survey study in the US, which used DSM criteria for diagnoses, the lifetime prevalence of PTSD (i.e., a PTSD diagnosis at any point prior to the study) was reported to be $7.8 \%$ among study participants who were representative of the general US population aged 15-54 years. ${ }^{2}$ In the National Comorbidity Survey replication, a nationally representative study of adults aged 18 years and older in the US, the lifetime prevalence was estimated to be $6.8 \% .{ }^{11}$ In a population-based survey study of young 
adults (aged 14-24 years) in Munich, the prevalence of lifetime PTSD was estimated to be lower, $1.3 \%$, and a 12-month prevalence of $0.7 \% .{ }^{12}$ Similarly, a general population survey study in Australia found the 12-month prevalence of PTSD to be $1.33 \% .{ }^{13}$ Using population-based data from six countries that were part of the European Study of the Epidemiology of Mental Disorders Survey, the 12-month prevalence of PTSD was estimated to be $1.1 \%{ }^{14}$ This prevalence estimate is consistent with the 12-month prevalence that has been reported across 11 countries that participated in the World Mental Health Surveys. ${ }^{15}$

It is widely held that PTSD is more common among women than men in the general population. ${ }^{2,16}$ Cross-sectional prevalence studies of the general population have consistently reported that PTSD is more prevalent among women than men. ${ }^{2}$ In one of the first population-based studies to examine gender-specific PTSD prevalence, Resnick et a ${ }^{17}$ used random digit dialing to obtain a sample of women residing in the US and found an overall prevalence of $12.3 \%$. Subsequent studies confirmed an elevated prevalence among women. In the National Comorbidity Survey study, the prevalence of PTSD was estimated to be 5\% among men and 10.4\% among women. ${ }^{2}$ Other population-based studies using insurance data in the US and population-based data from six European countries have provided evidence that the prevalence of PTSD is higher among women than men. ${ }^{14,16}$

With regard to age of onset for PTSD, population-based research has consistently shown that the majority of cases occur before age 40. Utilizing data from the National Comorbidity Survey replication in the US, Kessler et $\mathrm{al}^{11}$ found that $75 \%$ of PTSD cases in their sample occurred before age 40 . One study - a nationally representative sample of Englishspeaking residents of Ireland aged 18 years and older - found a slightly different pattern with $75 \%$ of PTSD cases occurring before age 49 years. ${ }^{18}$

One of two population-based studies that examined adjustment disorder found a prevalence of $<1 \%$ among five European countries, although this surprisingly low proportion was attributed to the limitations of the diagnostic tool used. ${ }^{19,20}$ However, a recent population-based study in Germany found the prevalence to be $\sim 0.9 \%$ among those with clinically significant impairment. ${ }^{21}$ Another study using the Danish national registries found that adjustment disorder was most commonly diagnosed in this population (incidence rate in adults $=97 / 100,000$ person-years; in children $=85 / 100,000$ person-years). Unspecified reactions to severe stress had the next highest incidence, followed by acute stress reaction and then PTSD. Importantly, this study also reported that in 2007, there was a relatively large increase in the frequency for all disorders, which highlights the dynamic nature of prevalence and incidence. ${ }^{22}$

With regard to the gender and age-of-onset distributions of stress disorders other than PTSD, a recent study that examined all five stress disorder diagnoses found a consistent disorder distribution by gender, in that for all five diagnoses women made up $\sim 60 \%$ of people with the diagnosis, with men only being $40 \% .{ }^{22}$ This was also consistent across adults and children. Age of onset was also found to be consistent across disorders. The highest proportion of all five stress diagnoses occurred in the late teens to early twenties with subsequent declines and a second slight increase in diagnoses beginning in the late twenties, followed by a gradual decrease as age increased. ${ }^{22}$

\section{Mental health sequelae of stress disorders}

Psychiatric comorbidity, particularly depression, commonly occurs with stress disorders, with the literature on this topic focusing primarily on PTSD to date. In fact, PTSD and depression symptoms so commonly co-occur that this combined symptomatology has been described as potentially one overarching posttraumatic symptom response that encompasses both disorders. ${ }^{23}$ This co-occurrence of PTSD and depression has been consistently demonstrated in the epidemiologic literature. In the National Comorbidity Survey in the US, men with PTSD had 6.9 times the odds of having a major depressive episode than men without PTSD (95\% confidence interval $[95 \% \mathrm{CI}]=4.4,11)$ and women with PTSD had 4.1 times the odds of a major depressive episode than women without PTSD $(95 \% \mathrm{CI}=3.1,5.4) .{ }^{2}$ The National Epidemiologic Survey on Alcohol and Related Conditions - a nationally representative study of adults aged 18 years and older in the US - found that those with PTSD had 2.7 times the odds of major depression than those without PTSD using cross-sectional data. ${ }^{24} \mathrm{~A}$ smaller epidemiologic study of young adults aged 21-30 years in Michigan found that those with PTSD had 2.8 times the rate of major depression using retrospective data reported during a baseline interview (95\% $\mathrm{CI}=1.6,5.0) .{ }^{25}$ However, in a prospective 5-year follow-up to this study, those with new-onset PTSD had 11.7 times the rate of major depression $(95 \% \mathrm{CI}=4.0,35) .{ }^{26}$ Recently, a population-based cohort study of Danes with stress diagnoses found that all five diagnoses had a similar cumulative incidence pattern for subsequent depression diagnoses, with a sharp increase in incidence in the year after diagnoses and then a more gradual increase in the next 14 years, with 
the incidence of depression at the end of 15-year follow-up between $20 \%$ and $25 \%$, markedly higher than the incidence in the comparison group without stress diagnoses. ${ }^{22}$

Another common stress disorder comorbidity is anxiety disorder. $^{2}$ It has been estimated that $\sim 13 \%$ of people with PTSD also have generalized anxiety disorder diagnoses. ${ }^{27}$ In the National Comorbidity Survey in the US, men with PTSD had 5.9 times the odds of generalized anxiety disorder than men without PTSD $(95 \% \mathrm{CI}=2.6,13)$. Women with PTSD have 2.8 times the odds of generalized anxiety disorder than women without PTSD $(95 \% \mathrm{CI}=3.1,5.4)$. Data from the National Epidemiologic Survey on Alcohol and Related Conditions in the US yielded similar results - those with PTSD had 4.3 times the odds of having any anxiety disorder than those without PTSD $(95 \% \mathrm{CI}=3.8,4.8) .{ }^{24}$ In a longitudinal cohort study of Danes with stress diagnoses, the cumulative incidence of anxiety disorders following stress disorder diagnoses increased steadily over the 15 -year follow-up period, ultimately reaching an incidence of $10 \%-15 \%$. This pattern was consistent across all five ICD-10 stress diagnoses and significantly higher than the incidence in the non-stressdiagnosed comparison group. ${ }^{22}$

Substance abuse and dependence, encompassing both alcohol abuse and dependence and drug abuse and dependence, is a third widely accepted comorbidity of stress disorders. ${ }^{2}$ In the National Comorbidity Survey, men with PTSD had 2.1 times the odds of alcohol abuse or dependence $(95 \% \mathrm{CI}=1.1,3.7)$ and 2.9 times the odds of drug abuse and dependence $(95 \% \mathrm{CI}=1.5,5.8)$ than men without PTSD. Among women, the odds of alcohol abuse and dependence for those with PTSD was $2.5(95 \% \mathrm{CI}=1.8,3.5)$ and the odds of drug abuse and dependence was 4.5 (95\% CI = $3.1,6.4$ ) compared to those without PTSD. ${ }^{2}$ In the National Epidemiologic Survey on Alcohol and Related Drugs, those with PTSD had 1.8 times the odds of any alcohol or drug use disorder than those without PTSD $(95 \% \mathrm{CI}=1.7,2.1) .{ }^{24} \mathrm{In}$ a recent longitudinal cohort study based on the Danish national healthcare and social registries, alcohol abuse and dependence incidence increased steadily among people with stress disorder diagnoses, ultimately reaching between $10 \%$ and $15 \%$ at the end of 15 years. For drug abuse and dependence, the overall incidence was lower with a shallow increase over the study period, reaching a cumulative incidence around 5\% at the end of the study period. ${ }^{22}$

\section{Physical health sequelae of stress disorders}

Stress disorders, PTSD in particular, have long been implicated in the etiology of various somatic disorders. ${ }^{5,28}$ Some somatic conditions, such as cancer, have been the subject of discussion in scientific publications during the past 7 decades and even longer in historical literature..$^{29-31}$ Despite this, and plausible biological mechanisms for this association, research findings had been conflicting with some studies providing evidence of an association and other studies not. The same is true for gastrointestinal (GI) disorders. Even though GI disorders have been examined as a possible outcome of PTSD for over 2 decades, ${ }^{32}$ evidence regarding the association has been mixed. Later, we review the literature for each of these important potential physical health outcomes of stress disorders in greater detail. Conversely, a stronger literature base for an association between PTSD and cardiovascular disease (CVD) has developed in recent years. PTSD has been consistently shown to be associated with CVD in predominantly male samples of US veterans ${ }^{33-36}$ and in the general population. ${ }^{37,38}$ However, this association has been examined for other stress disorders less frequently.

\section{PTSD and cancer risk}

As stated earlier, cancer as a consequence of PTSD had been the subject of discussion in scientific publications during the past 7 decades and even longer in historical literature. ${ }^{29-31}$ The literature on this association has been conflicting, however, with some studies providing evidence of an association and others not and many studies focusing on specific stressors rather than stress disorder diagnoses. In the Finnish Twin Study, acute stress events were associated with breast cancer. ${ }^{39}$ In Denmark, smoking-related malignancies among mothers were found to be weakly associated with loss of a child. ${ }^{40}$ However, other studies found no evidence of an association, ${ }^{31}$ including two population-based studies of stress and cancer. ${ }^{41-43}$ Recently, a population-based study using registry data from Western Australia found no increased incidence of any type of cancer among persons diagnosed with stress or anxiety disorders, compared with the general population. ${ }^{44}$ A cohort study in Denmark found an overall null association between PTSD and cancer diagnoses (standardized incidence ratio $[\mathrm{SIR}]=1.0,95 \% \mathrm{CI}=0.88,1.2) .{ }^{45}$ Near-null associations were also found between PTSD and all immune-related cancers $(\mathrm{SIR}=1.0,95 \% \mathrm{CI}=0.77,1.4)$; non-melanoma skin cancer $(\mathrm{SIR}=1.2,95 \% \mathrm{CI}=0.88,1.6)$; all smoking- and alcohol-related cancers ( $\mathrm{SIR}=0.99,95 \%$ $\mathrm{CI}=0.72,1.3)$; lung, bronchial and tracheal cancers (SIR $=1.3,95 \% \mathrm{CI}=0.73,2.0)$; colon cancer including cancer of the rectosigmoid junction $(\mathrm{SIR}=0.59,95 \% \mathrm{CI}=0.19$, $1.4)$; cancer at other common sites $(\mathrm{SIR}=1.1,95 \% \mathrm{CI}=$ $0.87,1.5)$; breast cancer $(\mathrm{SIR}=1.2,95 \% \mathrm{CI}=0.82,1.7)$ and uterine cancer $(\mathrm{SIR}=1.4,95 \% \mathrm{CI}=0.46,3.3) .{ }^{45}$ 


\section{PTSD and GI disorders}

GI disorders have been examined as a possible outcome of PTSD for over 2 decades. ${ }^{32}$ Research examining the association between PTSD and GI disorders has yielded mixed evidence, however, owing in part to methodological differences across studies. ${ }^{32}$ Two prospective studies of US veterans have documented an association. ${ }^{46,47}$ In contrast, a large prospective study of disaster survivors in the Netherlands found no association between self-reported PTSD and de novo medically documented combined GI disorders. ${ }^{48}$ In a large cohort of Danes who were diagnosed with a stress disorder from 1995 to 2011, the overall incidence rate of GI disorders was 1.8 times higher in the PTSD cohort than expected based on the rate in the general population $(95 \% \mathrm{CI}=1.7,2.0)$. No substantial association was found between PTSD and diverticula of the intestines ( $\mathrm{SIR}=1.1,95 \% \mathrm{CI}=0.83,1.5)$, and the magnitude of the remaining associations was variable. ${ }^{49}$

\section{Stress disorders and CVD}

In contrast to cancer outcomes, an association between PTSD and CVD has been well established in the literature. In the general population, people diagnosed with PTSD have 3.4 times the odds of heart failure than those without a PTSD diagnosis. ${ }^{37}$ Following the September 11, 2001, attacks, persons who developed PTSD had 1.7 times the risk of CVD than those without PTSD. ${ }^{38}$ A longitudinal cohort study using data on Danes diagnosed with stress disorders from 1995 to 2011 found moderate associations between PTSD and CVD, ranging from an SIR of $1.5(95 \% \mathrm{CI}=1.1,1.9)$ for myocardial infarction to $2.1(95 \% \mathrm{CI}=1.7,2.7)$ for venous thromboembolism. ${ }^{50}$ That study further examined a second stress disorder - adjustment disorder - which has received relatively less attention in the literature. The association between adjustment disorder and CVD in the same cohort was moderate, ranging from an SIR of $1.5(95 \% \mathrm{CI}=1.4$, 1.6) for myocardial infarction to $1.9(95 \% \mathrm{CI}=1.8,2.0)$ for venous thromboembolism. ${ }^{50}$

\section{Mortality following stress disorders Stress disorders and suicide}

Epidemiologic research on death from suicide as an outcome of trauma or stress disorders is limited. Although associations between trauma, stress disorders and suicidal behavior (e.g., suicide attempts) have been well documented in the literature, documentation of associations with suicide death is not. The distinction between studies of suicidal behavior and suicide death is an important one because these suicide events are more distinct than one may realize. For example, only $15 \%$ of people who make a suicide attempt go on to die by suicide. ${ }^{51}$ Later, we summarize the relevant literature on associations between individual stress disorder diagnosis and suicide.

\section{Acute stress reaction and suicide}

Only two studies have examined the association between acute stress reaction and suicide using epidemiologic samples. One cohort study conducted using data from the Danish national healthcare and social registries found a strong association between hospital-based acute stress reaction diagnoses and death from suicide. In adjusted analyses, those with acute stress reaction had 24 times the rate of suicide death than those without this diagnosis $(95 \% \mathrm{CI}=10,53){ }^{52}$ A case-control study conducted within this population found an association of $10(95 \% \mathrm{CI}=7.7,14) .^{53}$

\section{PTSD and suicide}

The vast majority of research on suicide outcomes following PTSD diagnoses has focused on suicidal ideation and suicide attempts; however, much of this research has been population based in contrast to the work done to date on the other stress disorders. In a recent cohort study of Danes with stress disorder diagnoses, the unadjusted association of hospitalbased PTSD diagnoses and death from suicide was $19(95 \%$ $\mathrm{CI}=7.6,46$ ). After adjustment for depression, anxiety and substance abuse diagnoses, the association decreased modestly - those with PTSD had 13 times the rate of suicide death than those without PTSD diagnoses $(95 \% \mathrm{CI}=4.3,42){ }^{52}$ In another study based on the Danish healthcare and social registries, those with PTSD had 5.3 times the rate of suicide than those without PTSD $(95 \% \mathrm{CI}=3.4,8.1) .{ }^{54}$

\section{Adjustment disorder and suicide}

There has been little epidemiologic research on the association between adjustment disorder and suicide, although studies in clinical samples provide evidence of an association. ${ }^{55,56}$ In a cohort study of Danes with stress disorder diagnoses from 1995 to 2011, the crude association between adjustment disorders and suicide was $18(95 \% \mathrm{CI}=15,21) .{ }^{52}$ After adjusting for baseline psychiatric confounders including hospital-based depression diagnoses, anxiety diagnoses and substance abuse and dependence diagnoses, we found a modest decrease in the observed association. Those with adjustment disorder had 12 times the rate of suicide compared to those without this diagnosis $(95 \% \mathrm{CI}=9.8,15) .{ }^{52}$ Another case-control study using epidemiologic registry data from Denmark documented an association of 19 for adjustment disorder and suicide $(95 \% \mathrm{CI}=17,21)$ adjusting only for the case-control 
matched factors. ${ }^{57}$ After additional adjustment for depression diagnoses, marital status and income, those with adjustment disorder diagnoses had 12 times the rate of suicide than those without these diagnoses $(95 \% \mathrm{CI}=11,14) .{ }^{57}$

\section{Unspecified stress diagnoses and suicide}

Similarly to acute stress reaction and adjustment disorder, only one study has examined the association between unspecified reaction to severe stress and suicide or suicidal behavior. In a large cohort study of Danes with stress diagnoses from 1995 to 2011, those with other reactions to severe stress had 29 times the rate of suicide and those with reactions to severe stress unspecified had 19 times the rate of suicide compared to those without these diagnoses. ${ }^{52}$

\section{Stress disorders and all-cause mortality}

Few studies have examined associations between stress disorders and all-cause mortality. PTSD was associated with a higher rate of all-cause mortality in large cohort studies of US veterans in both unadjusted and adjusted analyses, ${ }^{58-60}$ although one study found that this association was null after adjustment for relevant confounders. ${ }^{60}$ Only one study has examined all-cause mortality among people diagnosed with other stress disorders, a cohort study of Danes following persons' five ICD-10 stress disorder diagnoses from 1995 to $2011 .^{52}$ Unadjusted and adjusted associations that were similar in magnitude for all five stress diagnoses were found, with about double the rate of all-cause mortality among those with stress disorders than among the comparison group.

\section{Conclusion and future directions}

This review summarizes current literature on the longitudinal course of stress diagnoses (classified according to ICD-10) by examining their frequency and sequelae spanning mental health, physical health and causes of mortality domains. Understanding the occurrence and natural course of these diagnoses has critical implications for the development of secondary and tertiary prevention and intervention efforts. The first part of this review summarized what is known about the incidence and prevalence of stress disorders. From a public health perspective, the documentation of the prevalence and incidence of stress disorders has critical implications for intervention and prevention planning. This is especially important when considering the treatment of patients with these complex disorders in health care systems with limited resources (e.g., large physician caseload, short appointment windows).

Prevalence is impacted by many factors including changes in diagnostic practices or an increase in disease duration (i.e., how long people live with the disease or disorder). This is important because it highlights that cross-sectional studies of the prevalence of PTSD specifically should be interpreted with caution. The prevalence estimates reported in those studies may have just captured a temporary fluctuation in prevalence or may report a prevalence estimate that was accurate at the time but has since changed drastically. With regard to specific prevalence estimates, as summarized in this review, the US consistently has a higher documented prevalence of stress disorders, specifically PTSD, than other Westernized countries. Additional work is needed to examine the prevalence and incidence of stress disorders across populations and time and elucidate the causes of these differences.

In the second part of this review, we discussed the epidemiologic literature on three psychiatric disorders that are commonly comorbid with stress disorders - depression, anxiety disorders and substance abuse. This literature has largely focused on PTSD to date, with very limited research conducted on the other stress disorders. Results have been largely consistent across studies in that depression, anxiety disorders and substance abuse and dependence are frequently comorbid. Future population-based research should include examinations of all stress disorders and expand the scope of examined psychiatric comorbidities by including less common diagnoses (e.g., schizophrenia, bipolar disorder) and further elucidating the associations between stress disorders and subsyndromal comorbidity.

Next, we reviewed the epidemiologic literature on three important somatic consequences of hospital-based stress disorders - cancer, CVD and GI disorders. This line of research has important implications that call for tertiary prevention efforts with a focus on other somatic consequences of stress disorders. With regard to cancer, observed associations between PTSD and cancer would have had a major public health impact, given the ubiquity of PTSD and cancer and their costs to individuals and society. ${ }^{5,61}$ However, there are mixed results regarding evidence of associations, and thus, this remains an important area for future research. Future research should also expand in scope to examine additional stress diagnoses. With regard to CVD, there is literature to support consistent associations across stress disorder groups. This has important public health and clinical intervention and prevention implications given the ubiquity of these diagnoses. Finally, with regard to the association between PTSD and GI disorders, most studies have grouped GI disorders into one overall category for analysis, which may account for inconsistent findings; population-based studies that have examined GI 
disorders individually support this idea. ${ }^{49}$ Future research should continue to examine individual GI diagnosis and not overall categories and, as with the other somatic outcomes, expand this line of work to include stress disorders beyond PTSD. In addition, there may be other important somatic consequences of stress disorders that were not included in this review. A comprehensive review of all possible physical health outcomes of all stress disorders is beyond the scope of this work, but other physical health outcomes should be kept in mind when characterizing the full picture of the physical health sequelae of stress disorders. Finally, an unanswered question in the literature is whether it is traumatic or stressful events themselves that are associated with poorer physical health or stress disorders or both. Additional research is needed to elucidate these important and complex associations.

The last section of this review focused on mortality among people with stress disorders, both all-cause mortality and suicide-specific mortality. Stress disorders have been consistently shown to be associated with increases in all-cause mortality, and in some epidemiologic research, this could not be examined by somatic conditions. ${ }^{52}$ Future research should examine the mechanisms through which stress disorders result in an increased mortality risk. With regard to suicide mortality, stress disorders have displayed strong associations with suicide across studies, and this has been consistent across samples, time periods and varying methodology. Stress disorder diagnoses are strongly associated with death from suicide, even when adjusted for other psychiatric comorbidities. Stress disorders had been previously documented as predictors of suicidal behavior; however, only a small minority of people who make a suicide attempt and do not die go on to die by suicide. ${ }^{62}$ Therefore, persons who attempt suicide and who die by suicide are distinct populations. This work serves as a call for focused suicide intervention and prevention efforts among people with stress disorders, regardless of previous suicidal behavior.

Many epidemiologic studies do not examine the range of societal factors that may impact the clinical course of stress disorders. Social support and positive social networks are associated with decreased PTSD risk, and this relationship has been shown to be reciprocal in that increased PTSD symptomatology following trauma is also associated with social network deterioration. ${ }^{63,64}$ PTSD has also been associated with increased use of health care services and disability, ${ }^{65,66}$ which may have varying implications for patients in different countries with different health care systems and disability benefits. Finally, there is a strong body of research conducted among US veterans that shows that PTSD is associated with decreased employment, ${ }^{67,68}$ which may also have varying ramifications on stress disorder course depending on varying cross-national offerings and access of unemployment benefits, particularly in countries where health care benefits are closely tied to employment. All these factors may play a role in the path from stress disorder diagnoses to deleterious outcomes and serve as important contextual information to be mindful of when interpreting the findings of the studies reviewed herein.

Another issue to consider when reading this review is that it has focused on stress disorders classified according to ICD-10, but much of the literature in this area has been conducted in the US, where DSM criteria are used for psychiatric disorders. Ideally, both DSM diagnostic criteria and ICD diagnostic criteria should be describing the same underlying diagnostic phenomena, and studies utilizing either should be generalizable back to our understanding of that construct. However, psychiatry as a field lags behind other medical disciplines with regard to understanding the biology of many disorders, and diagnostic criteria may not align with true underlying disorder biology, resulting in predictor and outcome misclassification. Psychiatric diagnostic criteria represent the best the scientific community has to offer with regard to the observable representations of underlying etiology. However, if we had a full understanding of the biology of all psychiatric disorders and were able to diagnose and categorize them accordingly, classifications may be different than what we could glean from the currently used diagnostic criteria, which are based entirely on symptom observation and report. Thus, it is important to remember that much of what we know about the epidemiology of diagnoses included in the ICD-10 stress disorder category comes from DSMderived diagnoses.

In sum, stress disorders are a critical public health issue with potentially deleterious outcomes that have a significant impact on those living with these disorders, the health care system and society. It is only through an awareness of the impact of stress disorders that appropriate resources can be allocated to prevention and treatment. Future research should expand the work done to date beyond the examination of PTSD, so that the field may obtain a more complete picture of the impact all stress disorders have on the many people living with these diagnoses.

\section{Disclosure}

The author reports no conflicts of interest in this work. 


\section{References}

1. American Psychiatric Association. Diagnostic and Statistical Manual for Mental Disorders. 5th ed. Washington, DC: American Psychiatric Association; 2013.

2. Kessler RC, Sonnega A, Bromet E, Hughes M, Nelson CB. Posttraumatic stress disorder in the National Comorbidity Survey. Arch Gen Psychiatry. 1995;52(12):1048-1060.

3. Juel K, Sorensen J, Bronnum-Hansen H. Risk Factors and Public Health in Denmark: Summary Report. Copenhagen: National Institute of Public Health, University of Southern Denmark; 2007.

4. Schutt NM, Frederiksen ML, Helweg-Larsen K. Dating Violence in Denmark: A Nationwide Survey of Prevalence, Character, and Consequences of Exposure to Violence among 16-24 Year-Old. Copenhagen: Ministry of Gender Equality; 2008.

5. Kessler RC. Posttraumatic stress disorder: the burden to the individual and to society. J Clin Psychiatry. 2000;61(suppl 5):4-12.

6. Janca A, Ustun TB, van Drimmelen J, Dittmann V, Issac M. ICD-10 Symptom Checklist for Mental Disorders, Version 1.1. Geneva: Division of Mental Health, World Health Organization; 1994.

7. Crocq M-A, Crocq L. From shell shock and war neurosis to posttraumatic stress disorder: a history of psychotraumatology. Dialogues Clin Neurosci. 2000;2(1):47.

8. Munk-Jorgensen P, Kastrup M, Mortensen PB. The Danish psychiatric register as a tool in epidemiology. Acta Psychiatr Scand Suppl. 1993;370:27-32.

9. Maercker A, Brewin CR, Bryant RA, et al. Diagnosis and classification of disorders specifically associated with stress: proposals for ICD-11. World Psychiatry. 2013;12(3):198-206.

10. Peters L, Slade T, Andrews G. A comparison of ICD10 and DSMIV criteria for posttraumatic stress disorder. J Trauma Stress. 1999;12(2):335-343.

11. Kessler RC, Berglund P, Demler O, Jin R, Merikangas KR, Walters EE. Lifetime prevalence and age-of-onset distributions of DSM-IV disorders in the National Comorbidity Survey Replication. Arch Gen Psychiatry. 2005;62(6):593-602.

12. Perkonigg A, Kessler RC, Storz S, Wittchen H-U. Traumatic events and post-traumatic stress disorder in the community: prevalence, risk factors and comorbidity. Acta Psychiatr Scand. 2000;101(1):46-59.

13. Creamer M, Burgess P, McFarlane AC. Post-traumatic stress disorder: findings from the Australian National Survey of Mental Health and Well-being. Psychol Med. 2001;31(7):1237-1247.

14. Darves-Bornoz JM, Alonso J, de Girolamo G, et al. Main traumatic events in Europe: PTSD in the European study of the epidemiology of mental disorders survey. J Trauma Stress. 2008;21(5):455-462.

15. Karam EG, Friedman MJ, Hill ED, et al. Cumulative traumas and risk thresholds: 12-month PTSD in the World Mental Health (WMH) surveys. Depress Anxiety. 2014;31(2):130-142.

16. Breslau N, Davis GC, Andreski P, Peterson EL, Schultz LR. Sex differences in posttraumatic stress disorder. Arch Gen Psychiatry. 1997;54(11):1044-1048.

17. Resnick HS, Kilpatrick DG, Dansky BS, Saunders BE, Best CL. Prevalence of civilian trauma and posttraumatic stress disorder in a representative national sample of women. J Consult Clin Psychol. 1993;61(6):984-991.

18. Bunting BP, Murphy SD, O'Neill SM, Ferry FR. Lifetime prevalence of mental health disorders and delay in treatment following initial onset: evidence from the Northern Ireland Study of Health and Stress. Psychol Med. 2012;42(8):1727-1739.

19. Carta MG, Balestrieri M, Murru A, Hardoy MC. Adjustment disorder: epidemiology, diagnosis and treatment. Clin Pract Epidemiol Ment Health. 2009;5:15-30.

20. Casey P, Bailey S. Adjustment disorders: the state of the art. World Psychiatry. 2011;10(1):11-18.

21. Maercker A, Forstmeier S, Pielmaier L, Spangenberg L, Brähler E, Glaesmer H. Adjustment disorders: prevalence in a representative nationwide survey in Germany. Soc Psychiatry Psychiatr Epidemiol. 2012;47(11):1745-1752.
22. Gradus JL, Bozi I, Antonsen S, et al. Severe stress and adjustment disorder diagnoses in the population of Denmark. J Trauma Stress. 2014;27(3):370-374.

23. O'Donnell ML, Creamer M, Pattison P. Posttraumatic stress disorder and depression following trauma: understanding comorbidity. Am J Psychiatry. 2004;161(8):1390-1396.

24. Pietrzak RH, Goldstein RB, Southwick SM, Grant BF. Prevalence and axis I comorbidity of full and partial posttraumatic stress disorder in the United States: results from wave 2 of the National Epidemiologic Survey on Alcohol and Related Conditions. JAnxiety Disord. 2011;25(3):456-465.

25. Wilcox HC, Storr CL, Breslau N. Posttraumatic stress disorder and suicide attempts in a community sample of urban American young adults. Arch Gen Psychiatry. 2009;66(3):305-311.

26. Breslau N, Davis GC, Peterson EL, Schultz LR. A second look at comorbidity in victims of trauma: the posttraumatic stress disorder-major depression connection. Biol Psychiatry. 2000;48(9):902-909.

27. Kaufman J, Charney D. Comorbidity of mood and anxiety disorders. Depress Anxiety. 2000;12(S1):69-76.

28. Schnurr P, Jankowski M. Physical health and post-traumatic stress disorder: review and synthesis. Semin Clin Neuropsychiatry. 1999;4(4):295-304.

29. LeShan L. Psychological states as factors in the development of malignant disease: a critical review. J Natl Cancer Inst. 1959;22(1):1-18.

30. Reiche EMV, Nunes SOV, Morimoto HK. Stress, depression, the immune system and cancer. Lancet Oncol. 2004;5(10):617-625.

31. Chida Y, Hamer M, Wardle J, Steptoe A. Do stress-related psychosocial factors contribute to cancer incidence and survival? Nat Clin Pract Oncol. 2008;5(8):466-475.

32. Pacella ML, Hruska B, Delahanty DL. The physical health consequences of PTSD and PTSD symptoms: a meta-analytic review. J Anxiety Disord. 2013;27(1):33-46.

33. Kubzansky LD, Koenen KC, Spiro A, Vokonas PS, Sparrow D. Prospective study of posttraumatic stress disorder symptoms and coronary heart disease in the Normative Aging Study. Arch Gen Psychiatry. 2007;64(1):109-116.

34. Scherrer JF, Chrusciel T, Zeringue A, et al. Anxiety disorders increase risk for incident myocardial infarction in depressed and nondepressed Veterans Administration patients. Am Heart J. 2010;159(5):772-779.

35. Vaccarino V, Goldberg J, Rooks C, et al. Post-traumatic stress disorder and incidence of coronary heart disease. J Am Coll Cardiol. 2013;62(11):970-978.

36. Beristianos MH, Yaffe K, Cohen B, Byers AL. PTSD and risk of incident cardiovascular disease in aging veterans. Am J Geriatr Psychiatry. 2016;24(3):192-200.

37. Spitzer C, Barnow S, Volzke H, John U, Freyberger HJ, Grabe HJ. Trauma, posttraumatic stress disorder, and physical illness: findings from the general population. Psychosom Med. 2009;71(9):1012-1017.

38. Jordan HT, Miller-Archie SA, Cone JE, Morabia A, Stellman SD. Heart disease among adults exposed to the September 11, 2001 World Trade Center disaster: results from the World Trade Center registry. Prev Med. 2011;53(6):370-376.

39. Lillberg K, Verkasalo PK, Kaprio J, Teppo L, Helenius H, Koskenvuo M. Stressful life events and risk of breast cancer in 10,808 women: a cohort study. Am J Epidemiol. 2003;157(5):415-423.

40. Li J, Johansen C, Hansen D, Olsen J. Cancer incidence in parents who lost a child: a nationwide study in Denmark. Cancer. 2002;95(10):2237-2242.

41. Roberts FD, Newcomb PA, Trentham-Dietz A, Storer BE. Self-reported stress and risk of breast cancer. Cancer. 1995;77(6):1089-1093.

42. Schernhammer ES, Hankinson SE, Rosner B, et al. Job stress and breast cancer risk. Am J Epidemiol. 2004;160(11):1079-1086.

43. Johansen C, Olsen JH. Psychological stress, cancer incidence and mortality from non-malignant disease. Br J Cancer. 1997;75(1):144-148.

44. Kisely S, Crowe E, Lawrence D. Cancer-related mortality in people with mental illness. JAMA Psychiatry. 2013;70(2):209-217.

45. Gradus JL, Farkas DK, Svensson E, et al. Posttraumatic stress disorder and cancer risk: a nationwide cohort study. Eur J Epidemiol. 2015;30(7):563-568. 
46. Schnurr PP, Spiro A, Paris AH. Physician-diagnosed medical disorders in relation to PTSD symptoms in older male military veterans. Health Psychol. 2000;19(1):91-97.

47. Maguen S, Madden E, Cohen B, Bertenthal D, Seal K. Association of mental health problems with gastrointestinal disorders in Iraq and Afghanistan veterans. Depress Anxiety. 2014;31(2):160-165.

48. Dirkzwager AJE, van der Velden PG, Grievink L, Yzermans CJ. Disasterrelated posttraumatic stress disorder and physical health. Psychosom Med. 2007;69(5):435-440.

49. Gradus JL, Farkas DK, Svensson E, Ehrenstein V, Lash TL, Sørensen HT. Posttraumatic stress disorder and gastrointestinal disorders in the Danish population. Epidemiology. 2017;28(3):354-360.

50. Gradus JL, Farkas DK, Svensson E, et al. Associations between stress disorders and cardiovascular disease events in the Danish population. BMJ Open. 2015;5(12):e009334.

51. Maris RW, Berman AL, Silverman MM, editors. Introduction to the study of suicide. Comprehensive Textbook of Suicidology. New York: Guilford Press; 2000:3-25.

52. Gradus JL, Antonsen S, Svensson E, Lash TL, Resick PA, Hansen JG. Trauma, comorbidity, and mortality following diagnoses of severe stress and adjustment disorders: a nationwide cohort study. Am J Epidemiol. 2015;182(5):451-458.

53. Gradus JL, Qin P, Lincoln AK, et al. Acute stress reaction and completed suicide. Int J Epidemiol. 2010;39(6):1478-1484.

54. Gradus JL, Qin P, Lincoln AK, et al. Posttraumatic stress disorder and completed suicide. Am J Epidemiol. 2010;171(6):721-727.

55. Kryzhanovskaya L, Canterbury R. Suicidal behavior in patients with adjustment disorders. Crisis. 2001;22(3):125-131.

56. Greenberg WM, Rosenfeld DN, Ortega EA. Adjustment disorder as an admission diagnosis. Am J Psychiatry. 1995;153:759-761.

57. Gradus JL, Qin P, Lincoln AK, Miller M, Lawler E, Lash TL. The association between adjustment disorder diagnosed at psychiatric treatment facilities and completed suicide. Clin Epidemiol. 2010;2:23-28.
58. Boscarino JA. External-cause mortality after psychologic trauma: the effect of stress exposure and predisposition. Compr Psychiatry. 2006;47(6):503-514.

59. Boscarino JA. Posttraumatic stress disorder and mortality among US Army Veterans 30 years after military service. Ann Epidemiol. 2006; $16: 248-256$

60. Chwastiak LA, Rosenheck RA, Desai R, Kazis LE. Association of psychiatric illness and all-cause mortality in the National Department of Veterans Affairs Health Care System. Psychosom Med. 2010; 72(8):817-822.

61. Soerjomataram I, Lortet-Tieulent J, Parkin DM, et al. Global burden of cancer in 2008: a systematic analysis of disability-adjusted life-years in 12 world regions. Lancet. 2012;380(9856):1840-1845.

62. Maris RW, Berman AL, Silverman MM. Suicide attempts and methods. In: Maris RW, Berman AL, Silverman MM, editors. Comprehensive Textbook of Suicidology. New York, NY: The Guilford Press; 2000:284-310.

63. Kaniasty K, Norris FH. Longitudinal linkages between perceived social support and posttraumatic stress symptoms: sequential roles of social causation and social selection. J Trauma Stress. 2008;21(3):274-281.

64. Charuvastra A, Cloitre M. Social bonds and posttraumatic stress disorder. Annu Rev Psychol. 2008;59:301.

65. Deykin EY, Keane TM, Kaloupek D, et al. Posttraumatic stress disorder and the use of health services. Psychosom Med. 2001;63(5):835-841.

66. Koenen KC, Stellman SD, Sommer JF, Stellman JM. Persisting posttraumatic stress disorder symptoms and their relationship to functioning in Vietnam veterans: a 14-year follow-up. J Trauma Stress. 2008;21(1):49-57.

67. Zatzick DF, Marmar CR, Weiss DS, et al. Posttraumatic stress disorder and functioning and quality of life outcomes in a nationally representative sample of male Vietnam veterans. Am J Psychiatry. 2014;154(12):1690-1695.

68. Smith MW, Schnurr PP, Rosenheck RA. Employment outcomes and PTSD symptom severity. Ment Health Serv Res. 2005;7(2):89-101.
Clinical Epidemiology

\section{Publish your work in this journal}

Clinical Epidemiology is an international, peer-reviewed, open access, online journal focusing on disease and drug epidemiology, identification of risk factors and screening procedures to develop optimal preventative initiatives and programs. Specific topics include: diagnosis, prognosis, treatment, screening, prevention, risk factor modification,

Submit your manuscript here: https://www.dovepress.com/clinical-epidemiology-journa

\section{Dovepress}

systematic reviews, risk and safety of medical interventions, epidemiology and biostatistical methods, and evaluation of guidelines, translational medicine, health policies and economic evaluations. The manuscript management system is completely online and includes a very quick and fair peer-review system, which is all easy to use. 